\title{
High-density force myography: A possible alternative for upper-limb prosthetic control
}

\author{
Ashkan Radmand, PhD; Erik Scheme, PhD; Kevin Englehart, PhD* \\ Institute of Biomedical Engineering, University of New Brunswick, Fredericton, New Brunswick, Canada
}

\begin{abstract}
Several multiple degree-of-freedom upper-limb prostheses that have the promise of highly dexterous control have recently been developed. Inadequate controllability, however, has limited adoption of these devices. Introducing more robust control methods will likely result in higher acceptance rates. This work investigates the suitability of using highdensity force myography (HD-FMG) for prosthetic control. HD-FMG uses a high-density array of pressure sensors to detect changes in the pressure patterns between the residual limb and socket caused by the contraction of the forearm muscles. In this work, HD-FMG outperforms the standard electromyography (EMG)-based system in detecting different wrist and hand gestures. With the arm in a fixed, static position, eight hand and wrist motions were classified with $0.33 \%$ error using the HD-FMG technique. Comparatively, classification errors in the range of $2.2 \%-11.3 \%$ have been reported in the literature for multichannel EMG-based approaches. As with EMG, position variation in HD-FMG can introduce classification error, but incorporating position variation into the training protocol reduces this effect. Channel reduction was also applied to the HD-FMG technique to decrease the dimensionality of the problem as well as the size of the sensorized area. We found that with informed, symmetric channel reduction, classification error could be decreased to $0.02 \%$.
\end{abstract}

Key words: dynamic variation, electromyography, force myography, movement classification, myoelectric control, pattern recognition, position effect, prosthesis, prosthetic control, upper limb.

\section{INTRODUCTION}

Different types of prosthetic upper limbs (ULs), ranging from body-powered cable hooks to multifunction myoelectric prostheses, have been developed to offer people with amputation the ability to independently perform activities of daily living (ADLs). Each type accomplishes certain goals with regard to the user's expectations. However, many functional limitations in the prostheses decrease the acceptance rate by users. These limitations vary depending on the type of prosthesis. Discomfort caused by the harness, unappealing cosmetic appearance, and excessive motion or power required to operate bodypowered prostheses keep them from being an ideal solution [1-2]. Externally powered prostheses, especially myoelectric prostheses, can be more comfortable and usually provide a natural appearance, but still have deficiencies;

\footnotetext{
Abbreviations: $\mathrm{ADL}=$ activity of daily living, $\mathrm{DOF}=$ degree of freedom, EMG = electromyography, FMG = force myography, HD-FMG = high-density FMG, LDA = linear discriminant analysis, $\mathrm{P}=$ sensor pattern, Pos $=$ subject position, $\mathrm{S}$ $\mathrm{ADL}=$ simulated ADL, $\mathrm{SVM}=$ support vector machine, $\mathrm{UL}=$ upper limb.

*Address all correspondence to Kevin Englehart, PhD; Institute of Biomedical Engineering, University of New Brunswick, PO Box 4400, Fredericton, NB, Canada E3B5A3; 506458-7020; fax: 506-453-4867. Email: kengleha@unb.ca http://dx.doi.org/10.1682/JRRD.2015.03.0041
} 
they are expensive and provide less feedback to the user [3]. However, inadequate controllability, specifically lack of intuitive and dexterous control, is the main cause for the low rate of acceptance of externally powered prostheses. Improving the control strategies in different aspects, including accuracy, intuitiveness, and response time [4], may cause UL prostheses to experience a higher rate of acceptance [5].

UL prostheses can be controlled through different inputs. Surface electromyography (EMG) has long been one of the major neural control sources for powered UL prostheses. It contains useful information about the neuromuscular activity from which it originates, and it can be used to extract the user's intent [3]. Various EMG signal-processing methods have been used to extract movement intent. Conventional myoelectric control systems use an estimate of the signal magnitude (usually mean absolute value or root mean square) to quantify the intensity of muscle contractions as the control input. Although such control schemes have been widely used commercially, they are incapable of intuitively controlling more than one or two degrees of freedom (DOFs) $[3,6]$. Various forms of pattern recognition-based myoelectric control have been implemented to control multiple DOFs [7]. In this approach, a set of features containing spatial and temporal information about the acquired signals are extracted and form an input pattern to a classifier that determines the user's intended movement [3-4,8-9].

Pattern recognition algorithms have been widely investigated in terms of real-time implementation and performance [7,10-11], and a pattern recognition-based control system has recently been commercially deployed [12]. Low classification errors, in the range of 2.2 to 11.3 percent, have been reported for varying numbers (6 to 10) of wrist and hand movements using EMG pattern recognition techniques, such as linear discriminant analysis (LDA), artificial neural networks, and support vector machines (SVMs) [13-18].

Despite great successes in laboratory settings, several factors, such as variation in electrode placement [19-20], impedance, and the effect of socket loading, challenge pattern recognition-based myoelectric control in clinical settings. The position effect is also a challenging problem that has recently been the focus of several researchers [14-16]. The position effect refers to the degradation of myoelectric pattern recognition performance when the classifier is trained in one fixed position but is used in other positions or during dynamic activities [16]. Scheme et al. demonstrated that training the classifier in multiple positions reduces this degradation [14]. Chen et al. performed a similar study using data from participants with transradial amputation and supported the notion that training the classifier in multiple positions reduces the position effect [17]. In another study, Scheme et al. further showed that changing the limb position during both static and dynamic ADLs negatively affects myoelectric pattern recognition [15]. They proposed training the classifier with dynamic activities to reduce both of these effects. Boschmann and Platzner used a 96-channel highdensity electrode array and showed that training the classifier in multiple positions (three positions in their work) in combination with an increased number of EMG channels helped reduce the effect of limb position variation on classification accuracy [21].

The limitations of myoelectric control motivate investigation of alternative control inputs with a higher degree of robustness and accuracy. Many movements of the hand and wrist are controlled by muscles in the anterior and posterior compartments of the forearm known as extrinsic hand muscles. When the forearm muscles contract to move the hand, some muscles bulge outward while others recede inward from the surface. This results in pressure changes observable between the surface of the forearm skin and the socket. This pressure pattern may be used as the primary information source for prosthetic control [22-26]. The hypothesis is that the pressure patterns generated by various hand motions are distinct enough to differentiate the various motions from each other. Phillips and Craelius, who first proposed force myography (FMG), used pressure sensors to produce topographic maps of the pressure exerted against the hard prosthetic socket [22]. They used these pressure patterns to distinguish volitional finger flexion/extension. Yungher and Craelius then used an array of force sensors to measure pressure changes on the skin caused by muscular activity and a linear regression-based approach to accurately discriminate six different grasps with the arm in a fixed static position [23]. Li et al. recently used an array of 32 force-sensitive resistor sensors combined with an SVM classifier for finger-motion recognition based on pressure distribution maps [24]. They were able to accurately identify 17 different finger motions in within-session validation. Xiao and Menon prototyped an easy-to-use force sensor strap to extract the FMG signals from the proximal portion of the forearm [25]. They could classify, in real-time, six UL postures needed to complete a drinking task with an average overall error of 7.67 percent. 
Other groups have used pressure sensors in attempts to minimize the effects of pressure on EMG recording sites but not as the primary information source for control [27].

In this work, we use a technique referred to as highdensity FMG (HD-FMG) to distinguish the pressure patterns. HD-FMG measures changes in surface pressure caused by the displacement (i.e., the bulge) of the underlying muscle, movement of the bone, or even displacement of soft tissue to create a circumferential pressure map of the limb. HD-FMG extends previous FMG methods [22-25] by completely encircling the forearm with a custom, high-resolution grid of 126 pressure sensors. This advantage enables HD-FMG to accurately sense even small changes in the surface pressure map of the limb. Also, with greater coverage and resolution, HD-FMG requires no custom placement of sensors within the socket. HD-FMG uses standard pattern recognition algorithms to discriminate the patterns and determine the user's intent. In this study, we compared the performance of HD-FMG with that of previously reported pattern recognition-based EMG control approaches using an experimental protocol similar to those reported in the literature [13-18].

Previous work using FMG employed unrealistically ideal conditions, measuring static contractions in fixed positions. In real-world prosthetic use, however, a user must elicit contractions in a variety of positions and orientations and under different loading conditions. These conditions affect signal patterns and erode the accuracy of EMG pattern recognition methods [14-16]. In this work, we determined the effect of limb position on the accuracy of HD-FMG by conducting trials with static and dynamic variation in limb position. For EMG control, incorporating static and dynamic position variation into the training protocol can improve the resilience to position variation during use [14]. We used this methodology in this study to evaluate its potential for improvement with the HD-FMG method.

Finally, we investigated channel reduction when using HD-FMG. Reducing the number of pressure sensors mounted in the prosthetic socket is desirable for many reasons. As the number of sensors reduces, so does the number of control inputs along with the dimensionality of the classification problem and the computational complexity. Channel reduction also reduces hardware requirements, and so the cost of producing the pressure-sensing socket decreases. With fewer sensors, the sensorized area can be smaller, simplifying the fit within a socket. For channel reduction, however, even though the goal is to reduce the number of sensors, it is important to maintain a sufficient number of sensors to accurately measure the muscular displacement. Otherwise, the advantages of using a high-density configuration will be lost.

\section{METHODS}

\section{Population and Data Acquisition}

We collected pressure maps corresponding to eight classes of motion from 10 right-handed, healthy male subjects with intact ULs. Subjects ranged in age between 25 and 33 yr. The University of New Brunswick's Research Ethics Board approved all experiments.

The pressure maps were recorded using a highdensity grid of 126 (14 longitudinal rows $\times 9$ circumferential columns, with respect to the forearm) forcesensitive resistors $1 \times 1 \mathrm{~cm}$ in size that were custom made for this application by Smart Skin Technologies, Inc (Fredericton, New Brunswick, Canada). The array was mounted inside an adjustable prosthetic socket as shown in Figure 1(a). The flexible sensor grid covered the dominant forearm, centered at the position with the largest muscle bulk, with the sensors encircling it. Two zip ties, one at the top and one at the bottom of the socket, were used to adjust the socket size for each subject, and any socket gap was aligned with the ulna bone (Figures 1(b)-(c)). Because of the density and coverage of the grid, targeted placement of the sensors was not required. The 126 channels of pressure data were sampled at $20 \mathrm{~Hz}$ by a custom data-collection system. Data from the pressure sensors generate a pressure map image, composed of a single amplitude value for each of the 126 pixels for each reading.

\section{Experiments}

To study the performance of the HD-FMG technique, we compared the classification results with those of standard pattern recognition-based EMG control methods that have been reported in the literature [13-18], and followed a similar experimental protocol as that used by Radmand et al. [28]. Subjects were prompted to elicit contractions corresponding to eight classes of motion including wrist flexion/extension, wrist supination/pronation, pinch grip, power grip, hand open, and no movement. Each contraction was sustained for $3 \mathrm{~s}$, and a $3 \mathrm{~s}$ rest was given between subsequent contractions. Subjects were instructed to perform contractions at a moderate and repeatable force 


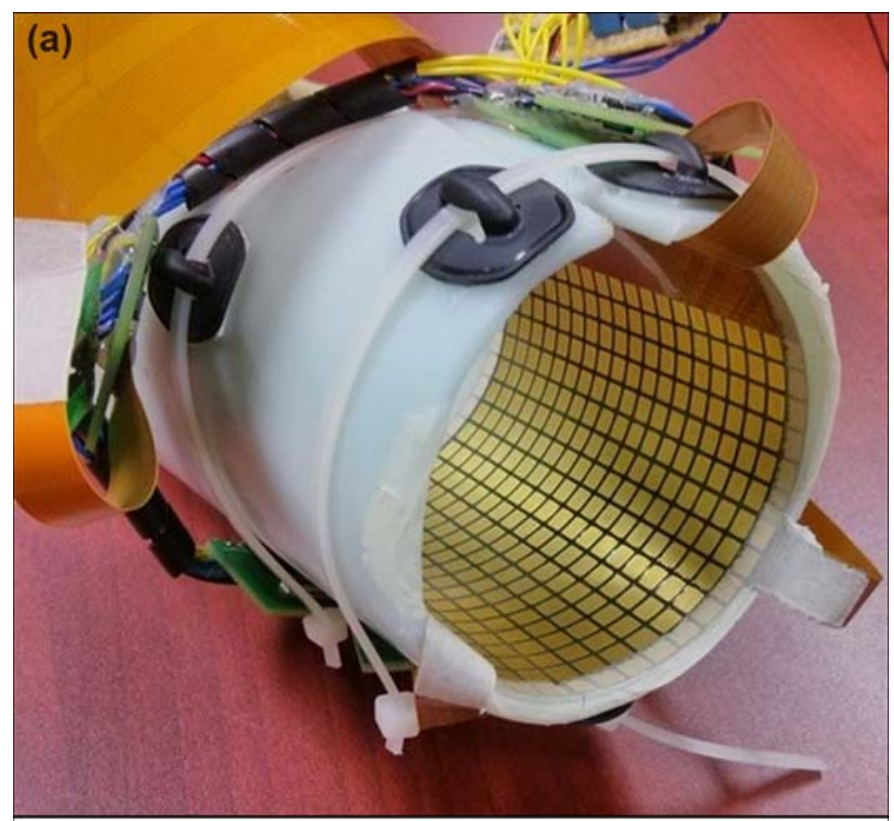

(b)
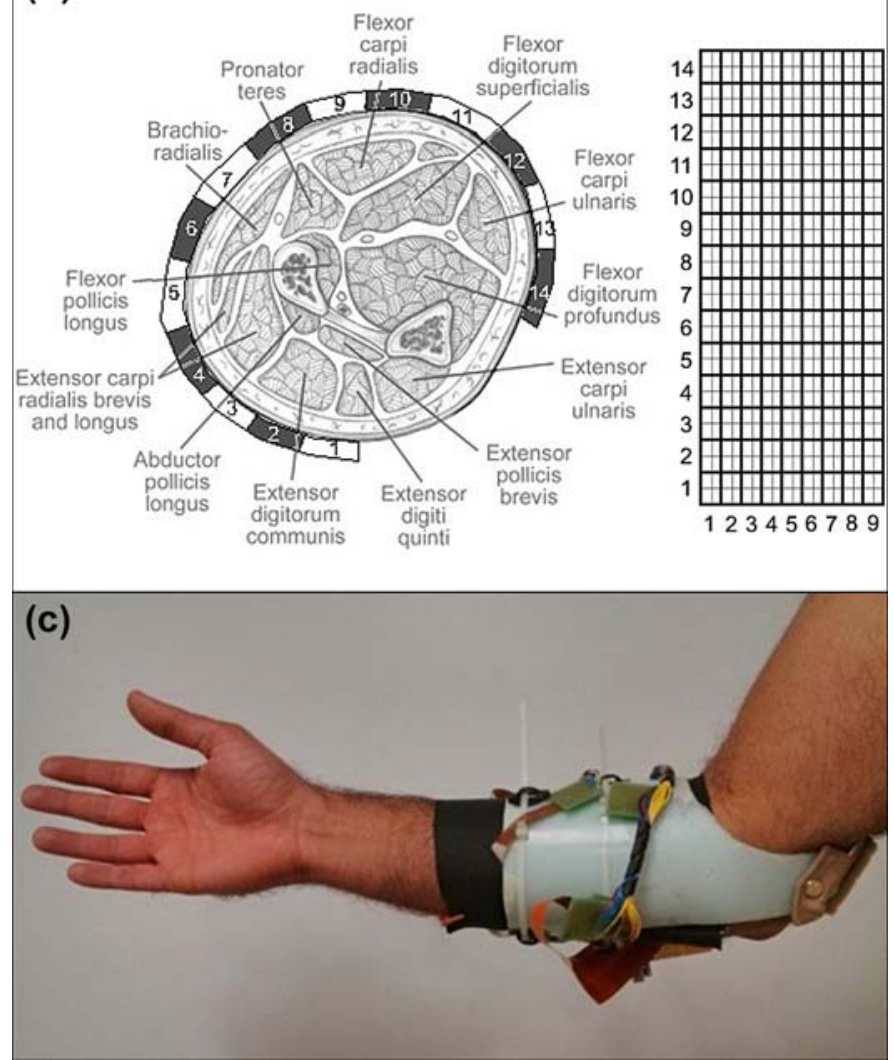

Figure 1.

(a) Adjustable pressure-sensing socket. Each $2 \times 2$ array of cells forms a single pressure sensor. (b) Sensor grid with its corresponding location on the muscles. (c) Placement of the socket with zip ties used to adjust socket size. level and given rest periods between trials to avoid fatigue. The data were collected while subjects were seated in an armchair, with the arm held in a fixed position, and the elbow resting on the chair's arm. Four sets of contractions were collected for each subject.

Then, to study the effect of limb position variation on the performance of the HD-FMG method, the same set of contractions was repeated during three sessions, each involving a different form of positional variation.

\section{Session 1: Static Positions}

Subjects sustained the motions while holding the arm in the eight static limb positions located on a plane parallel to the sagittal plane, passing through the humerus as shown in Figure 2. As first laid out in Radmand et al.

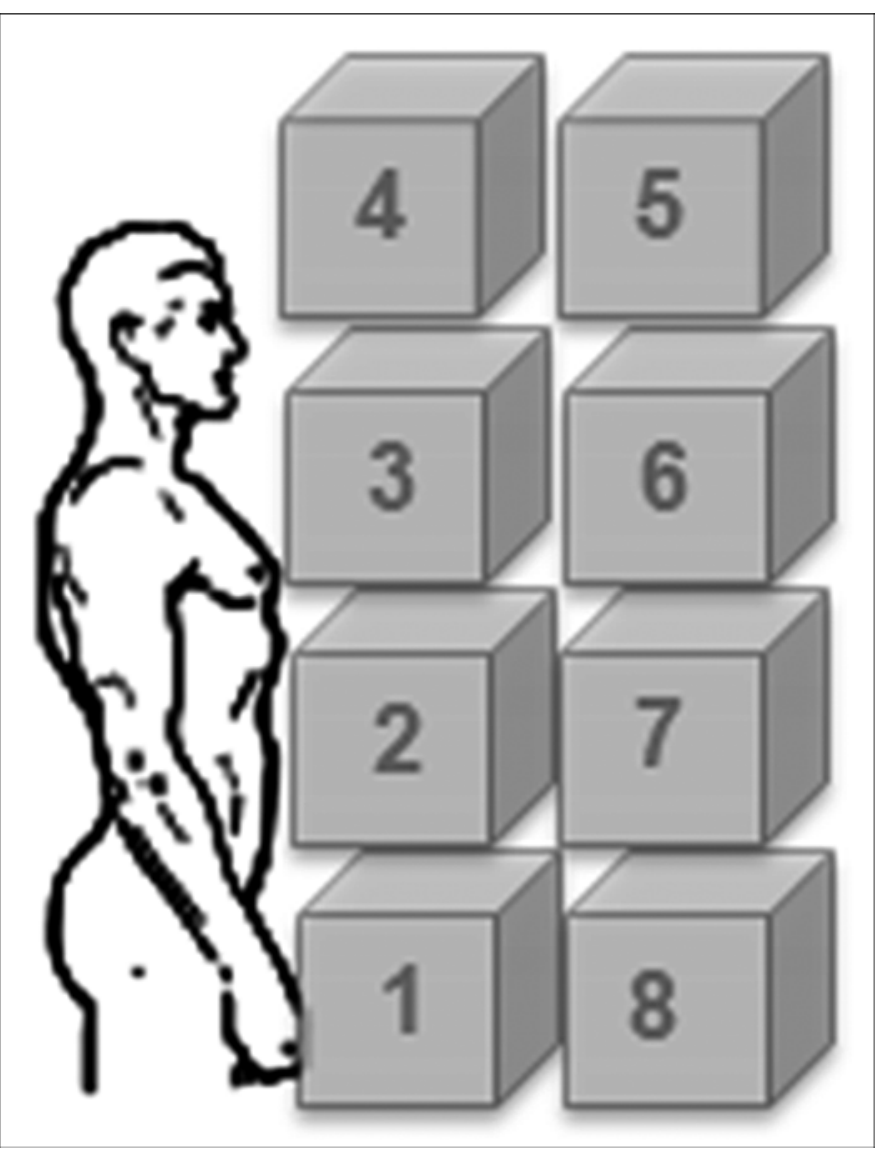

Figure 2.

Subjects were asked to perform four sets of contractions corresponding to eight classes of motion while holding their arm such that the hand was located in each of the eight static positions represented by the boxes numbered 1 through 8 . Reprinted with permission from Radmand et al. [28]. 
[28], "These positions cover the workspace in which most activities of daily living (ADL) are performed. To ensure that all subjects moved their arms to the same set of eight positions, the subjects were asked to stand in front of a white board, on which a grid of four cells corresponding to positions P1-P4 was drawn, and move the limb as if they want to reach the center of each cell. When data from all four of these positions were collected, the board was moved away from the subject to elicit the other four positions (P5-P8) and data were collected from those positions. Before each session, the distance from the board to the subject, the height of the board, and the spatial distribution of the cells were adapted for the height and reach of the subject.”

\section{Session 2: Activities of Daily Living}

A more meaningful assessment of the usability of a control system is its accuracy while a subject is performing ADLs, which are a specific subset of dynamic tasks identified by occupational therapists as meaningful functional activities. Subjects were asked to perform simulated ADLs (S-ADLs) while holding each of the eight classes of motion:

- S-ADL1: Subject position (Pos) 7 to Pos 3 (table top to drink),

- S-ADL2: Pos 1 to Pos 6 (neutral to table top), and

- S-ADL3: Pos 1 to Pos 5 (neutral to cupboard)

(Figure 3).

Contraction classes were held for $4 \mathrm{~s}$ during performance of S-ADLs, with $3 \mathrm{~s}$ interclass delays.

\section{Session 3: Dynamic Motion}

Executing all eight motions in every static position or during each S-ADL would be time-consuming for train- ing; having only one dynamic trial would take much less time. The subjects were asked to smoothly move the arm through a circular trajectory, starting from Pos 1 , through Pos 2 to 8, shown in Figure 4, while holding each of the classes of motion. Contraction classes were held for $15 \mathrm{~s}$ during dynamic motion performance, with $3 \mathrm{~s}$ interclass delays.

Four sets of contractions were collected for each of the static or dynamic positions, and the average duration of the experiment was approximately 120 min per subject.

\section{Data Processing and Classifier}

No specific data processing was performed on the pressure data; consequently, each decision was computed from an analysis window of $50 \mathrm{~ms}$ (due to the $20 \mathrm{~Hz}$ sampling rate). Moderate contraction intensities generally produced a pressure profile of sensor readings reasonably distributed between 0 and 1, with minimal clipping. These amplitudes were the only input to the classification stage.

To recognize the acquired pressure patterns of different motion classes, we used an LDA classifier [4] as a real-time control scheme for prosthetic control. This approach has been widely accepted because of its relative ease of implementation and high performance [11].

Fourfold cross-validation was used to test the performance of the HD-FMG technique. For each iteration, the data from three of the four repetitions of each motion were used for training, and the data from the fourth repetition was used for testing. The classification results reported represent the average across all four iterations.

\section{HD-FMG Channel Reduction}

In order to achieve the best performance, we equipped the pressure-sensing socket used for this work with
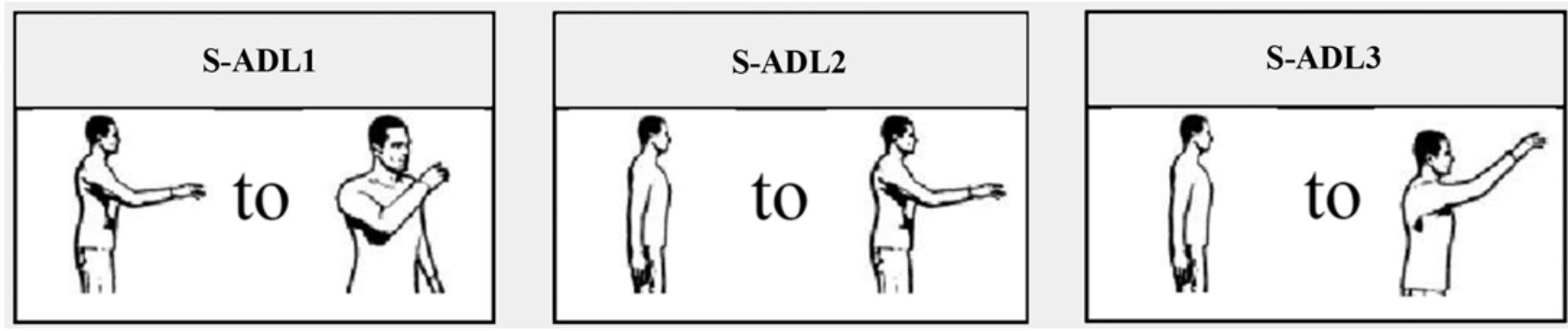

Figure 3.

Three simulated activities of daily living (S-ADL1 to S-ADL3) were completed while subjects were holding each of the classes of motion (figure modified from Scheme et al. [15] with permission). 


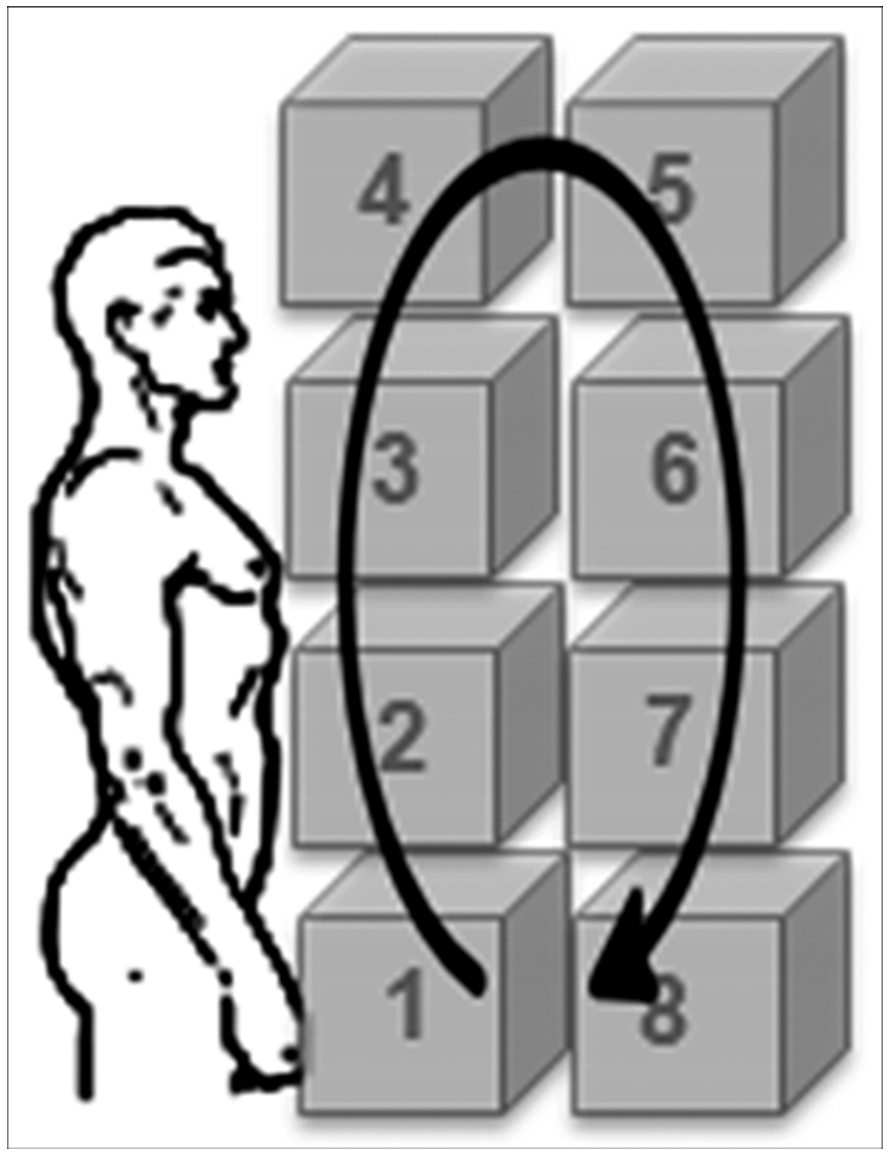

Figure 4.

Subjects were asked to smoothly move their arm through a circular trajectory.

126 pressure sensors, located in 14 rows and 9 columns, to completely encircle the limb and accurately monitor its shape. To investigate the optimal subset of channels and the effect of channel reduction on performance, we performed two rounds of channel reduction. We performed the first round of channel reduction over the sensor rows with all columns available, with the aim of reducing sensor density and hardware requirements. In order to avoid the need for accurate sensor placement inside the socket, we heuristically reduced the channels using the symmetrical patterns shown in Figure 5. The first pattern, which represents no reduction, was used as a baseline for comparison. In the next three patterns, every fourth, third, and second row, respectively, was left out. Pattern five represented a symmetric reduction of every second sensor in rows and columns. In the sixth pattern, we used only two separate groups of recording sites, covering the flexor and extensor muscles. The goal of this selection was to avoid placement of sensors on the top or bottom of the forearm to reduce any potential loading effects, which may cause changes in the socket pressure unrelated to the actual contractions.

A second round of channel reduction was performed over the sensor columns with the aim of reducing the length of the sensorized area within the socket. A brute-force method was applied, meaning that for each distinct number of sensor columns, the results were acquired and compared for every possible consecutive subset of the available columns. A constraint was applied to ensure that the selected columns were consecutive and that any resulting reduction effectively reduced the overall sensor dimensions.

\section{RESULTS}

\section{Pressure Maps}

Examples of the pressure maps acquired for each of the eight motion classes computed from a representative subject performing static contractions in a fixed position are shown in Figure 6. Although most subjects produced a general profile that resembled that of Figure 6, individual pressure maps exhibited distinct differences between subjects. In these pressure maps, the areas of low and high pressure are clearly visible for each motion, resulting in a distinct image for each motion.

\section{Hand Motion Classification}

Motion classification was performed for eight classes of motion, with the user's arm in a fixed static position. The results of applying HD-FMG for classification were compared with those of standard pattern recognition-based EMG control methods reported in the literature [13-18]. As shown in Figure 7, the mean overall error was 0.33 percent using pressure maps, while errors between 2.2 and 11.3 percent have been commonly reported using EMG signals [13-18].

The interclass confusion matrix for classification using the HD-FMG method is shown in Figure 8. Entries of the matrix represent the average accuracy on the diagonal and the average error everywhere else across all subjects and repetitions for the indicated class of motion. A perfect classification result would yield 100 percent on the diagonal and 0 percent everywhere else. Using the HD-FMG method, only slight confusion occurred (during one iteration, from one user) when discriminating the "hand open" motion from "no movement." 




P1



P2

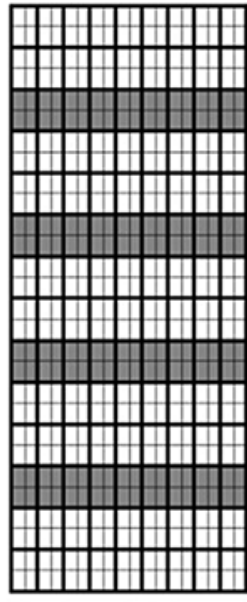

P3

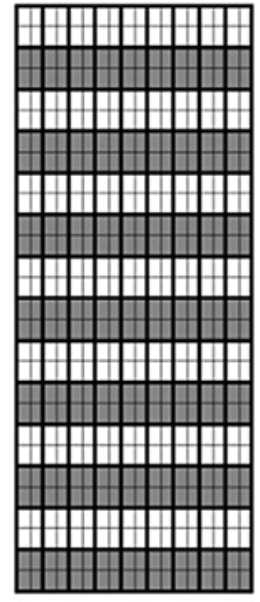

P4

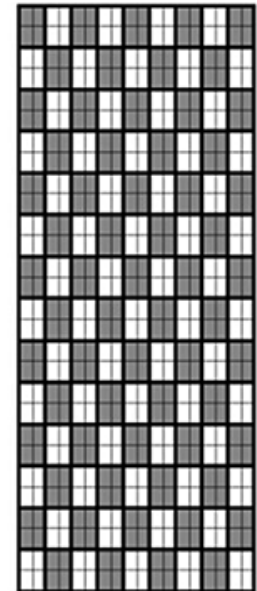

P5

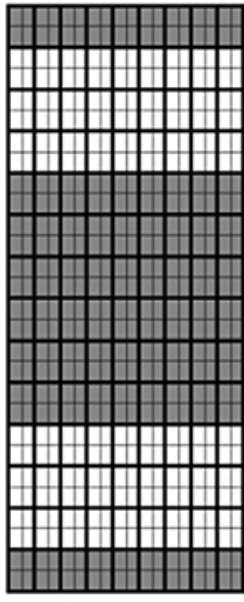

P6

Figure 5.

Heuristic channel selection patterns used for sensor density reduction. White cells are active sensors and gray cells are disabled sensors. $\mathrm{P}=$ sensor pattern.

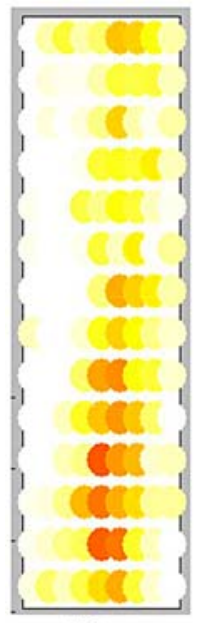

No

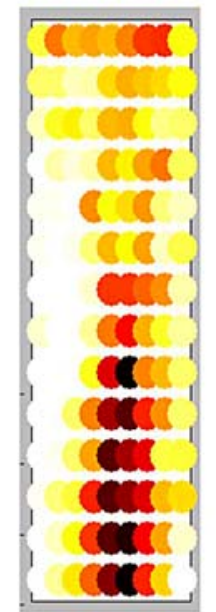

Wrist

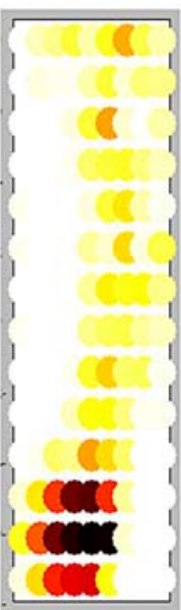

Wrist

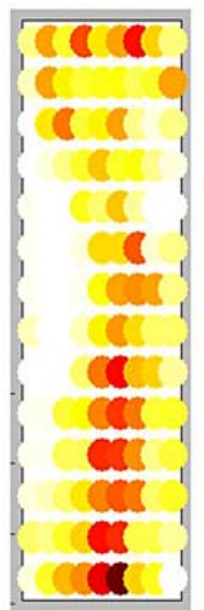

Wrist

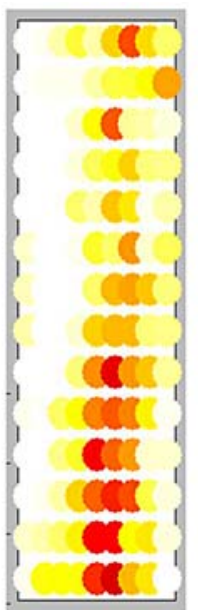

Wrist

\section{Movement Flexion}

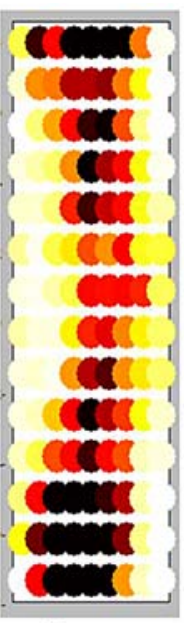

Power

Grip

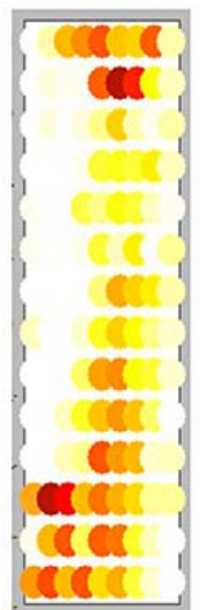

Pinch

Grip

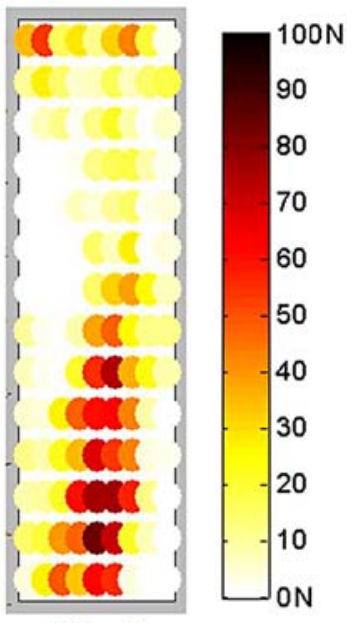

Hand

Open

Figure 6.

Examples of acquired pressure map images for the motion classes performed in a fixed static position (darker areas correspond to higher pressure).

\section{Limb Position Variation}

While HD-FMG showed a low level of error when the arm was in a fixed position, it is important to study the effect of limb position variation on its performance. Figure 9 shows how classification error increased when the classifier was trained in only one fixed static position and tested in either eight different static positions or while subjects were performing S-ADLs. These results show that, like EMG control, HD-FMG is susceptible to limb position variation as reported in Scheme et al. [15].

Training the classifier using data acquired in multiple positions or while subjects performed dynamic activity were two proposed potential solutions to the limb position 


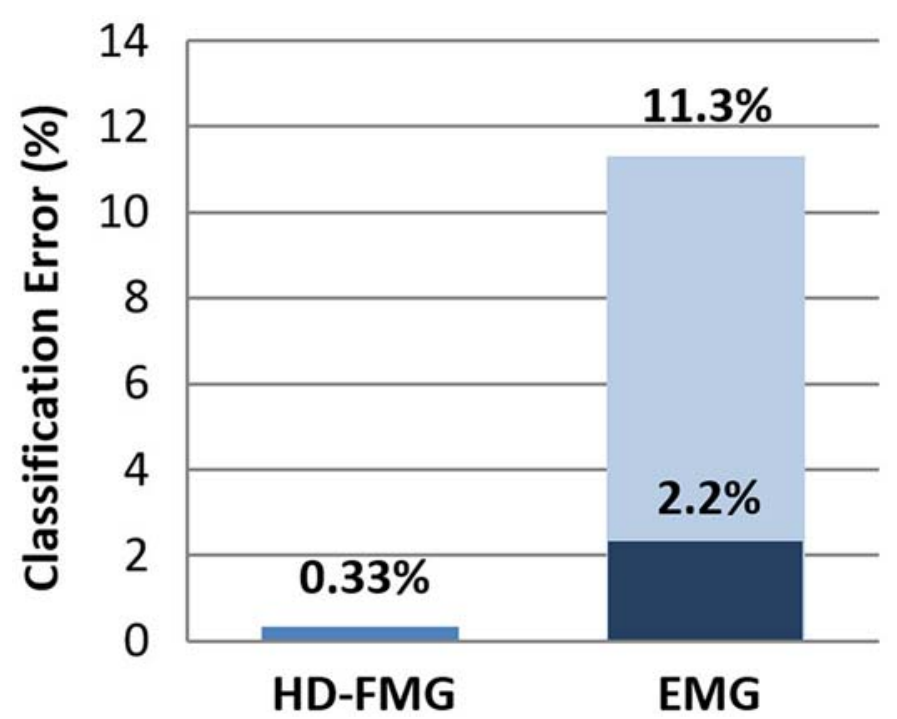

Figure 7.

Average classification errors of high-density force myography (HD-FMG) and electromyography (EMG) control methods for eight hand motion classes with arm in a fixed static position.

effect for classification accuracy with EMG signals [15]. We tried these proposed solutions to investigate whether they can be applied in a similar fashion to HD-FMG. Figure 10(a) shows the classification errors when varying numbers of positions (1-8) were included for training of the classifier. For each number of training positions, the average classification error of all subsets is shown. Figure 10(a) also shows the classification error when the classifier was trained while doing the dynamic activity described in the "Experiments" section (p. 445). The results show that dynamic training does not reduce the error for HD-FMG control. In each case, the classifier was tested with data from all eight positions. Figure 10(b) shows the classification errors when the classifier was tested with data from the three S-ADLs described in the "Experiments" section (p. 445).

HD-FMG multiposition training reduces the error when more static training positions are added, for both static test positions (Figure 10(a)) and S-ADLs (Figure 10(b)). Dynamic training does not reduce the error when testing with static positions and only marginally reduces error when testing with S-ADLs. We therefore recommend static multiposition training.

\section{Channel Reduction}

The results of applying row reduction using the patterns shown in Figure 5 are illustrated in Figure 11. These results suggest that while sensor pattern (P) 2, P3, and P4 are very close to $\mathrm{P} 1$ in terms of classification error, $\mathrm{P} 5$ significantly increases and P6 significantly decreases the error. It is important to note, however, that although significant differences were obtained, the effect size is minimal because all patterns resulted in very low error rates.

\begin{tabular}{|c|c|c|c|c|c|c|c|c|}
\hline & \multicolumn{8}{|c|}{ Classified Motion } \\
\hline & NM & WF & WE & WP & WS & CG & PG & HO \\
\hline NM & $97.65 \%$ & $0.00 \%$ & $0.00 \%$ & $0.00 \%$ & $0.00 \%$ & $0.00 \%$ & $0.00 \%$ & $2.35 \%$ \\
\hline WF & $0.00 \%$ & $100.00 \%$ & $0.00 \%$ & $0.00 \%$ & $0.00 \%$ & $0.00 \%$ & $0.00 \%$ & $0.00 \%$ \\
\hline WE & $0.00 \%$ & $0.00 \%$ & $100.00 \%$ & $0.00 \%$ & $0.00 \%$ & $0.00 \%$ & $0.00 \%$ & $0.00 \%$ \\
\hline WP & $0.00 \%$ & $0.00 \%$ & $0.00 \%$ & $100.00 \%$ & $0.00 \%$ & $0.00 \%$ & $0.00 \%$ & $0.00 \%$ \\
\hline WS & $0.00 \%$ & $0.00 \%$ & $0.00 \%$ & $0.00 \%$ & $100.00 \%$ & $0.00 \%$ & $0.00 \%$ & $0.00 \%$ \\
\hline CG & $0.00 \%$ & $0.00 \%$ & $0.00 \%$ & $0.00 \%$ & $0.00 \%$ & $100.00 \%$ & $0.00 \%$ & $0.00 \%$ \\
\hline PG & $0.00 \%$ & $0.00 \%$ & $0.00 \%$ & $0.00 \%$ & $0.00 \%$ & $0.00 \%$ & $100.00 \%$ & $0.00 \%$ \\
\hline HO & $0.38 \%$ & $0.00 \%$ & $0.00 \%$ & $0.00 \%$ & $0.00 \%$ & $0.00 \%$ & $0.00 \%$ & $99.62 \%$ \\
\hline
\end{tabular}

Figure 8.

Interclass confusion matrix for high-density force myography control method, averaged across all subjects and repetitions. CG $=$ chuck grip, $\mathrm{HO}=$ hand open, $\mathrm{NM}=$ no movement, $\mathrm{PG}=$ power grip, $\mathrm{WE}=$ wrist extension, $\mathrm{WF}=$ wrist flexion, $\mathrm{WP}=$ wrist pronation, $\mathrm{WS}=$ wrist supination. 
Figure 12 illustrates the classification errors for varying numbers of columns of sensors mounted in the prosthetic socket. Column reduction was performed twice, once with all rows available as the baseline and once with only the rows available in P6 as the best pattern selected in round one. For each number of columns, the classification errors of the best possible consecutive subset of columns and the average of all consecutive subsets of that size are shown. The results are shown for P1 and P6 in Figures 12(a) and (b), respectively, and the best subsets of columns for both patterns are compared in Figure 12(c).

These results showed that, on average, increasing the number of columns improved classification performance. A multivariate analysis of variance was completed on the results, with subject as a random factor and column combination as a fixed factor. The results showed, however, that for P1 and P6, the error when using three columns of

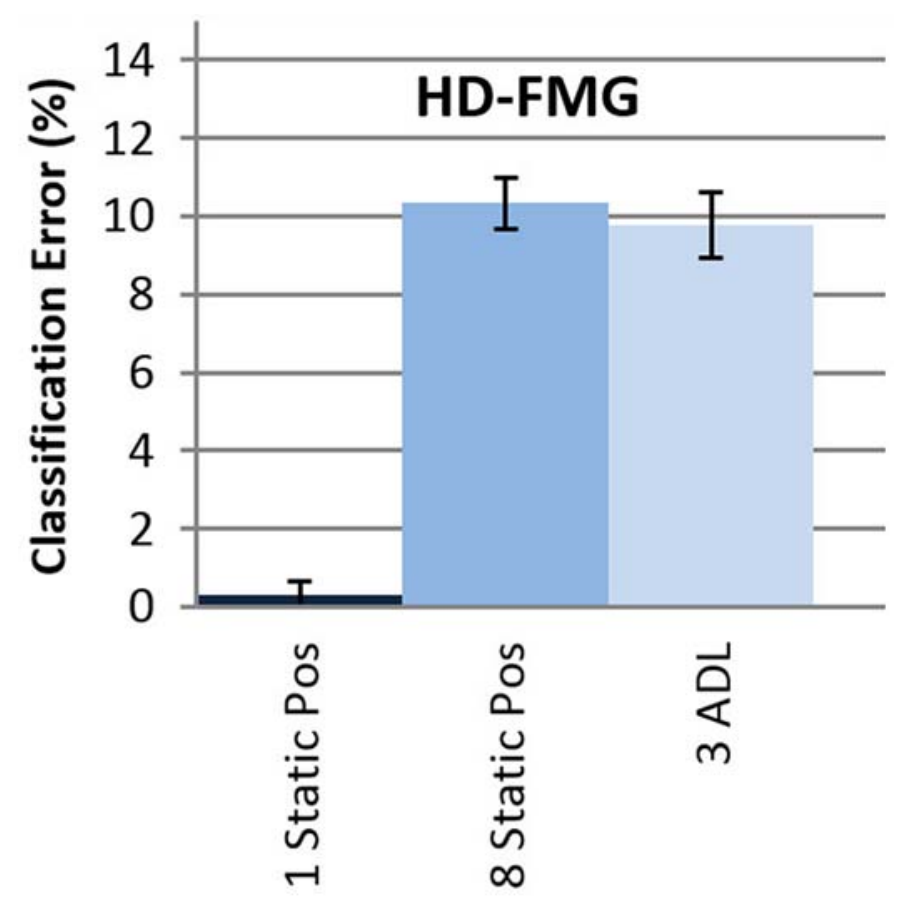

\section{Test Positions}

Figure 9.

Illustration of increase in classification error when a classifier that has been trained in only a fixed position (Pos) is tested during dynamic limb movements. Error bars indicate standard error across all subjects. ADL = activity of daily living, HD-FMG = high-density force myography. sensors was not significantly different from the results achieved when using all nine columns. Use of fewer than three columns of sensors, however, significantly increased error. Also, comparison of the best consecutive subsets for varying number of columns using rows of P1 and P6 showed that, when using more than four columns, P6 would result in lower classification error with fewer number of sensors than P1.

\section{DISCUSSION}

The results of hand motion classification illustrated in Figure 7 showed that the HD-FMG control technique is highly accurate in detecting different wrist and hand gestures. Compared to EMG, this method generated significantly lower classification errors. Using 126 force sensors for the HD-FMG technique, while comparing to studies that used only six to eight channels of EMG, may raise questions. However, the described HD-FMG system may provide a clinically and commercially viable approach, whereas a similarly high-density EMG-based system would likely not. Consequently, HD-FMG was compared with literature results consistent with the instrumentation of current commercially available pattern recognitionbased myoelectric control systems [12]. Furthermore, studies have shown that the use of more than eight channels of EMG does not significantly improve myoelectric classification accuracy above that reported here [29-33]. It would be preferable to directly compare HD-FMG and EMG signals and to possibly combine them, but the nature of the HD-FMG sensor instrumentation prohibited recording both signals simultaneously.

HD-FMG may also have some additional advantages. With the high resolution and full coverage of the socket, HD-FMG does not require targeted electrode location. The pressure sensors are very light and thin, making them comfortable and unobtrusive for the wearer. Existing EMG sensors, such as stainless steel electrodes, apply substantial and uneven pressure on the skin, often creating pain points or skin irritation. The pressure sensor components of the HD-FMG are not required to be in direct contact with the skin (they could be worn over thin clothing), minimizing any risk of skin reaction. Also, the performance of HDFMG relies on muscle physiology, which is relatively stable over short periods. While fatigue and sweat can change the characteristics of EMG, the physical dimensions of the limb do not change appreciably over moderate time periods. 
(a)

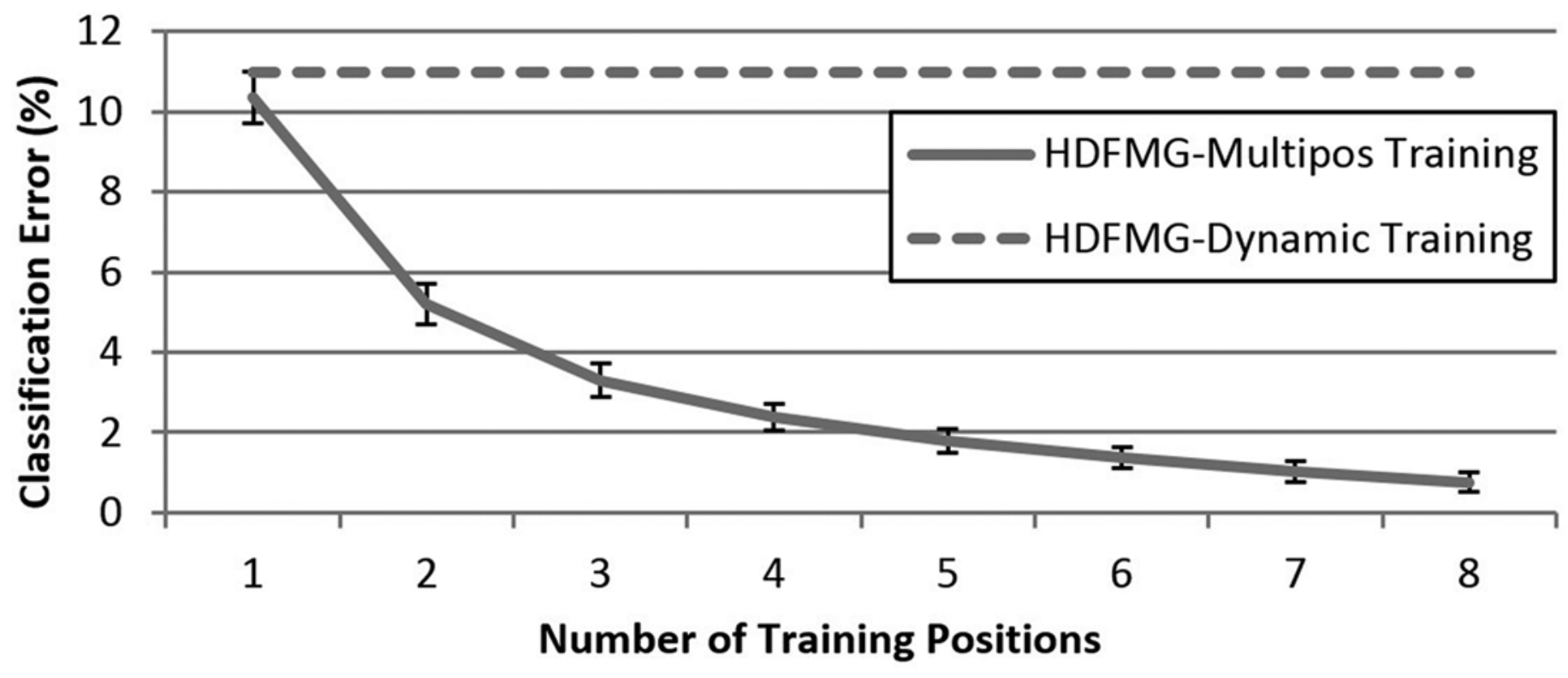

(b)

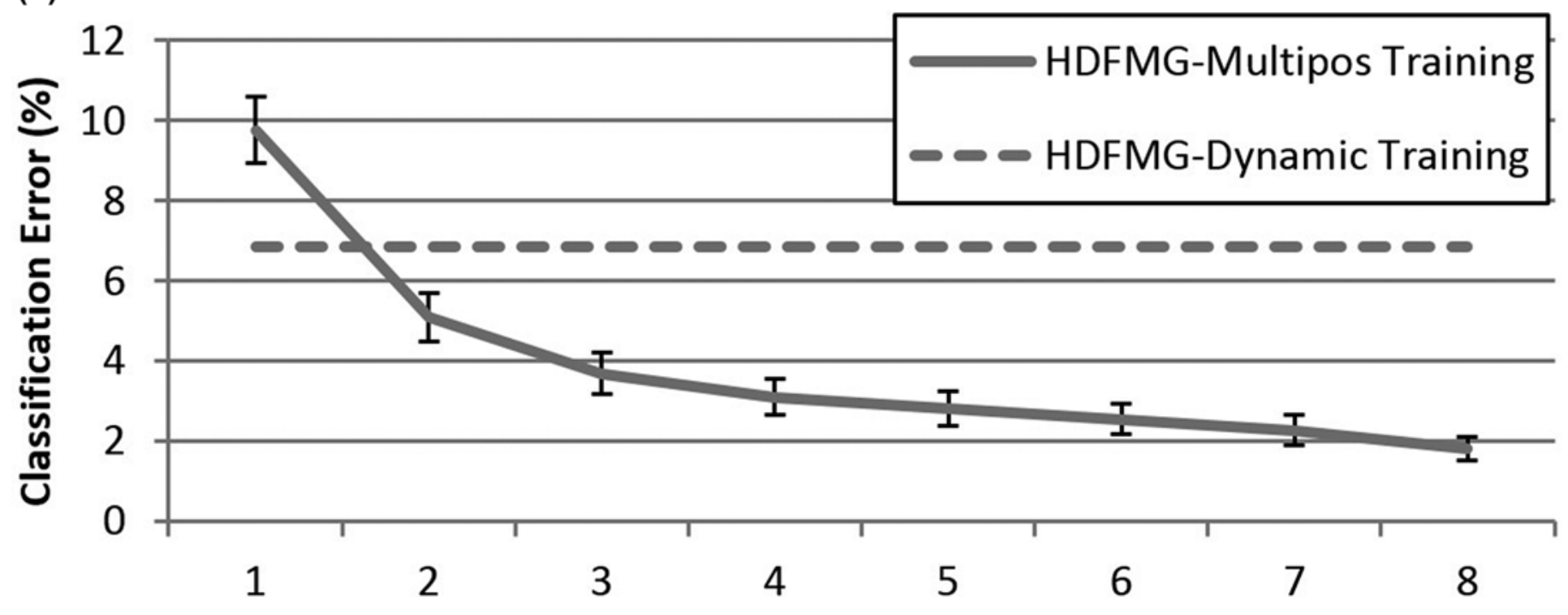

\section{Number of Training Positions}

Figure 10.

Illustration demonstrating that increasing the number of training positions reduced the classification error on average for a classifier that is tested (a) in eight static positions and (b) with three simulated activities of daily living. HDFMG = high-density force myography, Multipos = multiposition.

Electrode lift is another problem with EMG control because it changes the EMG signal characteristics, introducing substantial noise. This problem, however, does not exist with the proposed HD-FMG method, because contact is not needed; lift simply results in a 0 reading level, which may provide additional information if it is repeatable.

The analysis window of HD-FMG control (50 ms in this work) can be very short. This is because the proposed 


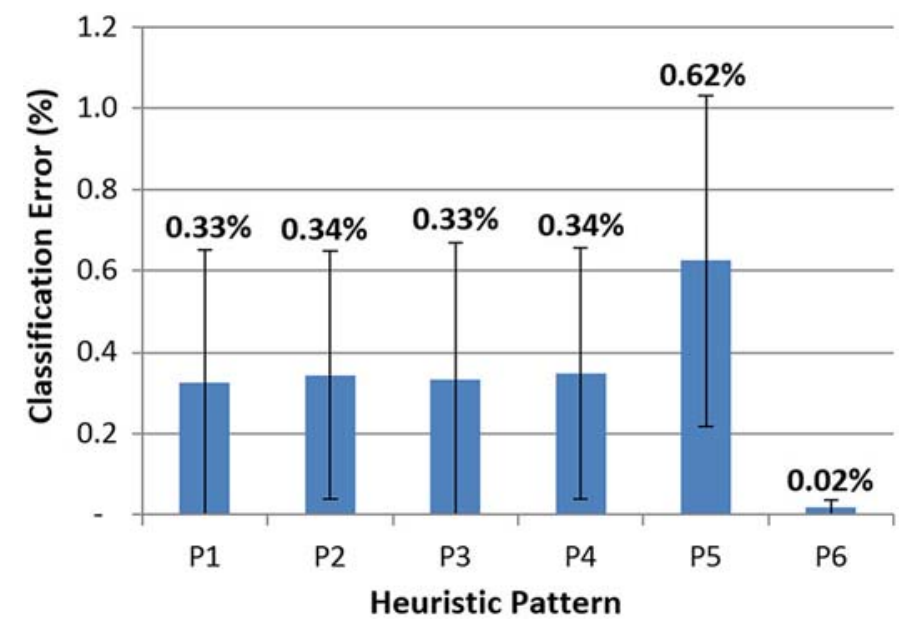

Figure 11.

Illustration demonstrating that reducing sensor density has minimal, but significant, effect on classification error. Error bars indicate standard error across all subjects. $\mathrm{P}=$ sensor pattern.

method does not explicitly rely on temporal information, and therefore, the length of the analysis window is only limited by the scanning frequency of the custom-acquisition hardware. Although not employed in this work, a majority vote postprocessing scheme could be employed within an acceptable window length to further improve the relative performance of the HD-FMG.

Figure 9 shows that limb position variation increases classification error in HD-FMG control. By training the classifier using data from multiple arm positions and including samples from different positions in the training data set, this error can be reduced, as shown in Figure 10. This method has been previously shown to be effective for resolving the position effect for EMG control [16]; it increases the repeatability between the training and testing data sets [28]. Another proposed solution to the position effect problem, dynamic training, was not very effective for HD-FMG control because muscle geometry changes as the arm moves in space.

The results of channel reduction illustrated in Figure 11 show that by monitoring only the flexor and extensor sides of the forearm, the error can be reduced considerably. This suggests that even minimal loading due to the weight of the socket may cause changes in the pressure patterns unrelated to the actual motions of the hand. We propose that in future work the loading surface at the top of the socket should be avoided. Also, the results of column reduction illustrated in Figure 12 suggest that the (a)

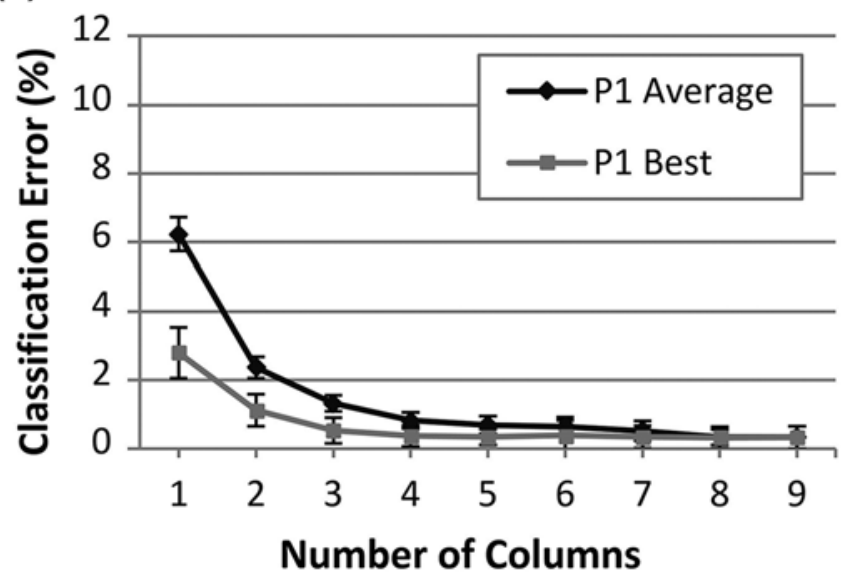

(b)

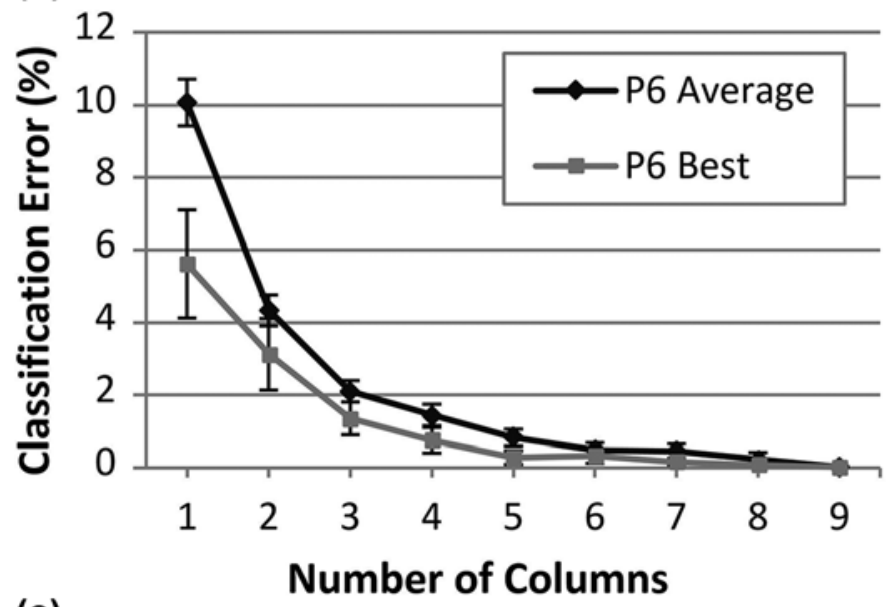

(c)

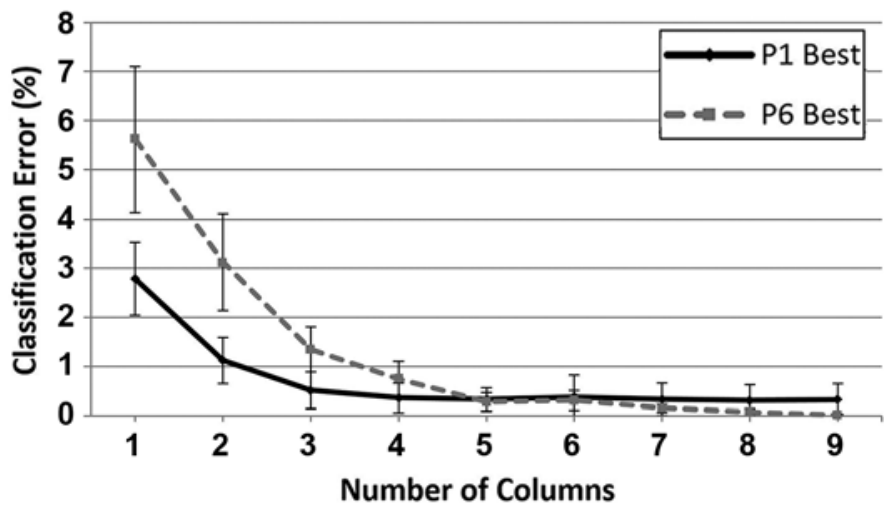

Figure 12.

Illustration demonstrating that increasing the number of columns improves the classification error on average when (a) all rows or (b) only P6 rows are available. (c) Comparison of the best subsets for varying number of columns using rows of P1 and P6. Error bars indicate standard error across all subjects. $\mathrm{P}=$ sensor pattern. 
size of the pressure sensing interface can be reduced to as few as three columns without any considerable drop in classification performance. Given the results in Figure 12(c), the use of five columns with only six rows, split evenly between the flexor and extensor sides, may provide a reasonable trade-off between classification accuracy and socket size and hardware requirement.

It is important to consider that this work represents a preliminary investigation of the HD-FMG approach, involving only nondisabled control subjects. Ongoing work, which will evaluate the performance of the approach with subjects with amputation, will require customization of the sensor boards to fit within custom sockets. It is possible that reduced muscle tone and surface deformations due to trauma or scar tissue could degrade the precision of the HDFMG approach. Nevertheless, these challenges also exist with EMG-based approaches, and we anticipate that HDFMG may still provide a reliable interface. Finally, HDFMG generates pressure maps that are similar to a digital image and uses pattern recognition algorithms to decode the user's intent. As images, the substantial body of shift/rotation tolerance in the image processing literature could potentially be employed in future work to accommodate sensor displacement or shift that may occur during or between uses of the device.

\section{CONCLUSIONS}

Although there is a significant body of research describing different techniques for EMG-based prosthetic control, their clinical robustness still needs improvement and novel methods of control that may improve robustness are of interest. In this work, we demonstrated that high-density monitoring of pressure patterns between the forearm and a socket can be used as a potential technique for prosthetic control. Compared with multichannel EMG control, HD-FMG demonstrates impressive accuracy in recognizing multiple movements of the wrist and hand. We also found that previously proposed methods to alleviate limb position effects on EMG control are applicable to HD-FMG control as well. In addition, we performed channel reduction over rows and columns of pressure sensors and demonstrated that, by monitoring only the flexor and extensor sides of the forearm and avoiding the loading surface at the top of the socket, HD-FMG performance increases. Future work will focus on validating the presented technique with subjects with amputation and developing sensor shift/rotation resilient techniques for robust, real-time pattern recognition-based control.

\section{ACKNOWLEDGMENTS}

\section{Author Contributions:}

Study concept and design: A. Radmand, E. Scheme, K. Englehart. Acquisition of data: A. Radmand.

Analysis and interpretation of data: A. Radmand, E. Scheme.

Drafting of manuscript: A. Radmand.

Critical revision of manuscript for important intellectual content:

A. Radmand, E. Scheme, K. Englehart.

Statistical analysis: A. Radmand.

Study supervision: A. Radmand, E. Scheme, K. Englehart.

Administrative, technical, or material support: E. Scheme, K. Englehart. Obtained funding: K. Englehart.

Financial Disclosures: The authors have declared that no competing interests exist. We are thankful to Smart Skin Technologies, Inc, Fredericton, New Brunswick, Canada, for manufacturing the experimental pressure sensors for the study. Smart Skin, Inc, did not have any involvement in the study design; data collection, analysis, or interpretation; or writing or submission of this article.

Funding/Support: This material was based on work supported by the Natural Sciences and Engineering Research Council of Canada (Discovery Grant 217354-10 and Engage Grant).

Additional Contributions: Thanks to Smart Skin Technologies, Inc (Fredericton, New Brunswick, Canada) for providing the force sensor arrays. In particular, thanks to Dr. Ian McKelvey for his contributions to the design of sensor electronics.

Institutional Review: The University of New Brunswick's Research Ethics Board approved all experiments. All participants gave informed consent.

Participant Follow-Up: The authors do not plan to inform participants of the publication of this study. However, participants have been encouraged to check the study Web site for updated publications.

\section{REFERENCES}

1. Millstein SG, Heger H, Hunter GA. Prosthetic use in adult upper limb amputees: A comparison of the body powered and electrically powered prostheses. Prosthet Orthot Int. 1986;10(1):27-34. [PMID:3725563]

2. Kitayama I, Matsuda M, Nakajima S, Sawamura S, Ninomiya H, Furukawa H. Improvement of control cable system of trans-humeral body-powered prostheses. Prosthet Orthot Int. 1999;23(2):123-29. [PMID:10493139]

3. Merletti R, Parker P. Electromyography: Physiology, engineering, and noninvasive applications. Hoboken (NJ): John Wiley \& Sons, Inc; 2004.

4. Englehart K, Hudgins B. A robust, real-time control scheme for multifunction myoelectric control. IEEE Trans 
Biomed Eng. 2003;50(7):848-54. [PMID:12848352]

http://dx.doi.org/10.1109/TBME.2003.813539

5. Biddiss E, Chau T. Upper-limb prosthetics: Critical factors in device abandonment. Am J Phys Med Rehabil. 2007; 86(12):977-87. [PMID:18090439]

http://dx.doi.org/10.1097/PHM.0b013e3181587f6c

6. Scott RN, Parker PA. Myoelectric prostheses: State of the art. J Med Eng Technol. 1988;12(4):143-51.

[PMID:3057209]

http://dx.doi.org/10.3109/03091908809030173

7. Oskoei MA, Hu H. Myoelectric control systems: A survey. Biomed Signal Process Control. 2007;2(4):275-94. http://dx.doi.org/10.1016/j.bspc.2007.07.009

8. Hudgins B, Parker P, Scott RN. A new strategy for multifunction myoelectric control. IEEE Trans Biomed Eng. 1993;40(1):82-94. [PMID:8468080]

http://dx.doi.org/10.1109/10.204774

9. Hargrove L, Losier Y, Lock B, Englehart K, Hudgins B. A real-time pattern recognition based myoelectric control usability study implemented in a virtual environment. In: 2007 29th Annual International Conference of the IEEE Engineering in Medicine and Biology Society; 2007 Aug 2226; Lyon, France. New York (NY): IEEE Press; 2007. p. 4842-45.

10. Micera S, Carpaneto J, Raspopovic S. Control of hand prostheses using peripheral information. IEEE Rev Biomed Eng. 2010;3:48-68. [PMID:22275201] http://dx.doi.org/10.1109/RBME.2010.2085429

11. Scheme E, Englehart K. Electromyogram pattern recognition for control of powered upper-limb prostheses: State of the art and challenges for clinical use. J Rehabil Res Dev 2011; 48(6):643-59. [PMID:21938652]

http://dx.doi.org/10.1682/JRRD.2010.09.0177

12. COAPT Engineering Company [Internet]. Chicago (IL): COAPT Engineering; 2014. Available from: http://www.coaptengineering.com/

13. Crawford B, Miller K, Shenoy P, Rao RP. Real-time classification of electromyographic signals for robotic control. In: Proceedings of the Twentieth National Conference on Artificial Intelligence AAAI 2005; 2005 Jul 9-13; Pittsburgh, PA. Palo Alto (CA): AAAI Press; 2005. p. 523-528.

14. Scheme E, Fougner A, Stavdahl Ø, Chan AD, Englehart K. Examining the adverse effects of limb position on pattern recognition based myoelectric control. In: 2010 Annual International Conference of the IEEE Engineering in Medicine and Biology; 2010 Aug 31-Sep 4; Buenos Aires, Argentina. New York (NY): IEEE Press; 2010. p. 6337-40.

15. Scheme E, Biron K, Englehart K. Improving myoelectric pattern recognition positional robustness using advanced training protocols. In: 2011 Annual International Conference of the IEEE Engineering in Medicine and Biology
Society; 2011 Aug 30-Sep 3; Boston, MA. New York (NY): IEEE Press; 2011. p. 4828-31.

16. Fougner A, Scheme E, Chan AD, Englehart K, Stavdahl Ø. Resolving the limb position effect in myoelectric pattern recognition. IEEE Trans Neural Syst Rehabil Eng. 2011; 19(6):644-51.

17. Chen L, Geng Y, Li G. Effect of upper-limb positions on motion pattern recognition using electromyography. In: 2011 4th International Congress on Image and Signal Processing (CISP); 2011 Oct 15-17; Shanghai, China. New York (NY): IEEE Press; 2011. p. 139-42.

18. Oweis RJ, Rihani R, Alkhawaja A. ANN-based EMG classification for myoelectric control. Int J Med Eng Inform. 2014;6(4):365-80.

http://dx.doi.org/10.1504/IJMEI.2014.065442

19. Biron K, Englehart K. EMG pattern recognition adaptation. In: XVIII Congress of the International Society for Electrophysiology and Kinesiology; 2010 Jun 16-19; Aalborg, Denmark; Victoria, BC (Canada): ISEK; 2010. p. 63.

20. Young AJ, Hargrove LJ, Kuiken TA. Improving myoelectric pattern recognition robustness to electrode shift by changing interelectrode distance and electrode configuration. IEEE Trans Biomed Eng. 2012;59(3):645-52.

[PMID:22147289]

http://dx.doi.org/10.1109/TBME.2011.2177662

21. Boschmann A, Platzner M. Reducing the limb position effect in pattern recognition based myoelectric control using a high density electrode array. In: 2013 ISSNIP Biosignals and Biorobotics Conference: Biosignals and Robotics for Better and Safer Living (BRC); 2013 Feb 18-20; Rio de Janeiro, Brazil. New York (NY): IEEE Press; 2013. p. 1-5.

22. Phillips SL, Craelius W. Residual kinetic imaging: A versatile interface for prosthetic control. Robotica. 2005;23(03): 277-82. http://dx.doi.org/10.1017/S0263574704001298

23. Yungher D, Craelius W. Discriminating 6 grasps using force myography of the forearm. In: 2007 Northeast American Society of Biomechanics (NEASB) Conference; 2007 Mar 30-31; College Park, MD; 2007.

24. Li N, Yang D, Jiang L, Liu H, Cai H. Combined use of FSR sensor array and SVM classifier for finger motion recognition based on pressure distribution map. J Bionic Eng. 2012;9(1):39-47. http://dx.doi.org/10.1016/S1672-6529(11)60095-4

25. Xiao ZG, Menon C. Towards the development of a wearable feedback system for monitoring the activities of the upper-extremities. J Neuroeng Rehabil. 2014;11(1):2. [PMID:24397984] http://dx.doi.org/10.1186/1743-0003-11-2

26. Wininger M, Kim N-H, Craelius W. Pressure signature of forearm as predictor of grip force. J Rehabil Res Dev. 
2008;45(6):883-92. [PMID:19009474]

http://dx.doi.org/10.1682/JRRD.2007.11.0187

27. Fougner A, Saether M, Stavdahl O, Kyberd P, Blum J. Cancellation of force induced artifacts in surface EMG using FSR measurements. In: Proceedings of the 2008 MyoElectric Controls/Powered Prosthetics Symposium; 2008 Aug 13-15; Fredericton, New Brunswick, Canada. The University of New Brunswick; 2008.

28. Radmand A, Scheme E, Englehart K. A characterization of the effect of limb position on EMG features to guide the development of effective prosthetic control schemes. In: 2014 36th Annual International Conference of the IEEE Engineering in Medicine and Biology Society (EMBC); 2014 Aug 26-30; Chicago, Illinois. New York (NY); IEEE Press; 2014. p. 662-67.

29. Hargrove LJ, Englehart K, Hudgins B. A comparison of surface and intramuscular myoelectric signal classification. IEEE Trans Biomed Eng. 2007;54(5):847-53. [PMID:17518281] http://dx.doi.org/10.1109/TBME.2006.889192

30. Nagata K, Ando K, Magatani K, Yamada M. Development of the hand motion recognition system based on surface EMG using suitable measurement channels for pattern recognition. In: 2007 29th Annual International Conference of the IEEE Engineering in Medicine and Biology Society; 2007 Aug 22-26; Lyon, France. New York (NY): IEEE Press; 2007. p. 5214-17.

31. Kvas G, Velik R. A filter approach for myoelectric channel selection. In: 2008 6th IEEE International Conference on
Industrial Informatics; 2008 Jul 13-16; Daejeon, Korea. New York (NY): IEEE Press; 2008. p. 1437-40.

32. Shibanoki T, Shima K, Tsuji T, Otsuka A, Chin T. A quasioptimal channel selection method for bioelectric signal classification using a partial Kullback-Leibler information measure. IEEE Trans Biomed Eng. 2013;60(3):853-61.

[PMID:22752103]

http://dx.doi.org/10.1109/TBME.2012.2205990

33. Geng Y, Zhang X, Zhang YT, Li G. A novel channel selection method for multiple motion classification using highdensity electromyography. Biomed Eng Online. 2014;13: 102. [PMID:25060509] http://dx.doi.org/10.1186/1475-925X-13-102

Submitted for publication March 9, 2015. Accepted in revised form September 23, 2015.

This article and any supplementary material should be cited as follows:

Radmand A, Scheme E, Englehart K. High-density force myography: A possible alternative for upper-limb prosthetic control. J Rehabil Res Dev. 2016;53(4):443-56. http://dx.doi.org/10.1682/JRRD.2015.03.0041

ORCID: Kevin Englehart, PhD: 0000-0003-4525-1121



